\title{
INFLUÊNCIA DO MANEJO HIGIÊNICO-SANITÁRIO NA INFECÇÃO POR HELMINTOS GASTRINTESTINAIS EM SUÍNOS DE GRANJAS TECNIFICADAS E DE SUBSISTÊNCIA ABATIDOS NA REGIÃO METROPOLITANA DE RECIFE E ZONA DA MATA DO ESTADO DE PERNAMBUCO, BRASIL
}

\author{
S. d'Alencar ${ }^{1}$, M.P.O. Farias ${ }^{1}$, E.O. Rosas ${ }^{1}$, M.M. de Lima ${ }^{2}$, L.C. Alves ${ }^{1}$, M.A.G. Faustino ${ }^{1}$
}

${ }^{1}$ Universidade Federal Rural de Pernambuco, Departamento de Medicina Veterinária, R. do Machado 1012/402B, CEP 52120-250, Recife, PE, Brasil. E-mail: dalencar_ale@yahoo.com

\section{RESUMO}

\begin{abstract}
Objetivou-se com este trabalho analisar a influência do manejo higiênico-sanitário nos níveis de infecção por parasitos gastrintestinais em suínos de granjas tecnificadas e de subsistência abatidos na Região Metropolitana de Recife e Zona da Mata do Estado de Pernambuco. Para isso, o processo de abate de suínos em três abatedouros inscritos no sistema de inspeção estadual foi acompanhado durante o período de julho de 2008 a maio de 2009 , num total de 447 suínos oriundos de 11 propriedades de municípios do Estado de Pernambuco, sendo oito granjas tecnificadas e três de subsistência. Amostras fecais foram coletadas da ampola retal no abatedouro e avaliadas pelos métodos de Gordon \& Whitlock e Roberts \& O’Sullivan. Realizou-se a aplicação de um questionário investigativo para conhecer a situação das granjas. Para o OPG a positividade foi de 2,7\% (12/447), sendo 2,5\% (11/447) para ovos tipo Strongyloidea e 0,2\% (1/447) para Trichuris sp. Para a coprocultura, 5,5\% (19/349) foram positivas para helmintos, sendo 2,9\% (10/349) para Hyostrongylus sp., 0,3\% (1/349) para Trichostrongylus sp., 2,0\% (7/349) para Oesophagostomum spp. e infecção mista por Hyostrongylus sp. e Oesophagostomum spp. em 0,3\% (1/349). Associação significativa $(p<0,05)$ com a ocorrência de helmintos foi obtida para as variáveis "gênero de helminto", "alimentação", "assistência veterinária", "quarentena", "comércio de animais vivos", "anti-helmíntico", "fonte de água", "tratamento de água", "qualidade de higiene das baias", "limpeza das instalações" e "conhecimento de verminose e manchas leitosas", com percentuais de positividade significativamente mais elevados entre os animais das granjas de subsistência.
\end{abstract}

PALAVRAS-CHAVE: Helmintose suína, Oesophagostomum sp., suinocultura, exame coproparasitológico, monitoria em abatedouro.

\section{ABSTRACT}

INFLUENCEOF HYGIENEMANAGEMENTONGASTROINTESTINAL HELMINTHINFECTION IN PIGS IN METROPOLITAN RECIFE AND THE COASTAL ZONE OF THE STATE OF PERNAMBUCO, BRAZIL. The aim of the present study was to analyze the influence of management systems at intensive high-tech farms and subsistence farms on the prevalence of swine gastrointestinal helminthes in metropolitan Recife and the coastal zone of the state of Pernambuco, Brazil. For such, the slaughtering process at three slaughterhouses registered with the state inspection system was followed up between July 2008 to May 2009, totaling 447 pigs from 11 properties (8 high-tech farms and 3 subsistence farms). Fecal samples were collected and the parasitological examinations were carried out using the modified McMaster technique and coproculture for identification of larvae. A questionnaire was administered to determine the management condition of each farm. Strongyloidea eggs were present in $2.5 \%(11 / 447)$ of the pigs, and $0.2 \%(1 / 447)$ were positive for Trichuris sp. Coproculture revealed that $5.5 \%$ (19/349) were positive for helminthes, $2.9 \%(10 / 349)$ for Hyostrongylus sp., $0.3 \%$ (1/349) for Trichostrongylus sp., 2.0\% (7/349) for Oesophagostomum sp., and $0.3 \%(1 / 349)$ with mixed infection by Hyostrongylus and Oesophagostomum sp. The occurrence of helminthes was significantly associated $(\mathrm{p}<0.05)$ with helminth gender, feeding, veterinary care, quarantine, live animal trade, anti-helminth agents, water supply, water treatment, hygiene

${ }^{2}$ Universidade Federal Rural de Pernambuco, Departamento de Biologia, Unidade Acadêmica de Serra Talhada, Serra Talhada, PE, Brasil. 
quality of stalls, dung removal frequency and knowledge regarding worms and milk spots. The percentages of positivity were significantly higher among animals on the subsistence farms.

KEY WORDS: Swine helminthiasis, Oesophagostomum sp., copro-parasitological examination, slaughterhouse monitoring.

\section{INTRODUÇÃO}

A suinocultura nacional ocupa o quinto lugar entre os maiores produtores de carne suína, contando com rebanho estimado de 35,2 milhões de cabeças. Atualmente, a região Nordeste possui o segundo maior rebanho de suínos do Brasil, com 6,7 milhões de cabeças, o que representa $18,77 \%$ do plantel de suínos nacional (IBGE, 2007).

Considerando o emprego de recursos tecnológicos, uma grande variação entre os sistemas de criação é observada. As criações em escala industrial empregam conhecimentos de genética, reprodução, nutrição, imunologia, e tratamentos anti-helmínticos no manejo diário para a obtenção de bons índices de produtividade (ROPPA, 1998; FAO, 2009). A suinocultura de subsistência possui interferência direta na vida da população que a produz, tendo uma importância social e econômica expressiva para os estados da região Nordeste, região na qual mais da metade da população depende diretamente do meio rural (SILVA FiLHA, 2007).

Em todas as fases de exploração suinícola, os animais estão expostos a parasitoses que produzem efeitos deletérios, influentes na capacidade produtiva dos rebanhos (Formiga et al., 1980). Os animais mais acometidos são os suínosjovens de seis semanas a seis meses, nos quais a ação parasitária é mais evidente e danosa. Animais adultos também são portadores destes helmintos e, geralmente, apresentam a forma subclínica da doença. Desta maneira, os animais infectados contaminam o ambiente, água, alimentos e fômites (Toma et al., 2003). Os prejuízos estão relacionados ao retardo na produção e aos custos com tratamento profilático e curativo e, em casos extremos, a morte dos animais.

A prevalência e importância econômica das parasitoses variam notavelmente em dependência do sistema de manejo, características dos alojamentos, medidas higiênico-sanitárias, localização geográfica da exploração eidade do suíno. Todas estas variáveis influenciam nos requerimentos básicos dos estágios de vida de livre dos parasitos, e nos mecanismos de transmissão e na resposta imune dos hospedeiros frente aos diferentes parasitos (LeITE et al., 2000; MORA, 2000).

A intensificação da produção de suínos tem permitido melhorar as práticas higiênico-sanitárias na exploração, tanto as destinadas para que os suínos tenham menor contato com seus dejetos (piso apropriado, limpeza e desinfecção rotineira dos alojamentos), como as que evitam um possível contato com os portadores, como o desmame precoce, separação dos animais por idade, quarentena de animais recém-adquiridos, práticas de "todos dentro, todos fora". Tudo isto tem sido importante para uma redução da diversidade de parasitas presentes nas granjas, ao evitar o desenvolvimento de muitos deles. Estas variações têm conduzido a uma notável modificação na epidemiologia das parasitoses de suínos (MORA, 2000).

As monitorias sanitárias são formas de constatar, qualificar e quantificar o nível sanitário de populações de suínos para determinada doença ou infecção. Dentre os tipos de monitoria sanitária, a monitoria patológica em abatedouro é uma das mais importantes fontes de informações para avaliação da situação da saúde de sistemas de produção de suínos (SONCINI; MADUREIRA JÚNIOR, 1998), sendo uma de suas aplicações mais frequentes, dentre outras, o estudo de prevalência de doenças, a identificação das causas mais frequentes de condenação em abatedouros e comparação de tipos de sistemas de produção (Kunz et al., 2003).

Sendo assim, objetivou-se, neste estudo, analisar a influência do manejo higiênico-sanitário nos níveis de infecção por parasitos gastrintestinais em suínos de granjas tecnificadas e de subsistência abatidos na Região Metropolitana de Recife e Zona da Mata do Estado de Pernambuco, Brasil.

\section{MATERIAL E MÉTODOS}

O trabalho foi realizado em três abatedouros inscritos no sistema de inspeção estadual cuja agência reguladora é a Agência de Defesa e Fiscalização Agropecuária de Pernambuco (ADAGRO), localizados na Região Metropolitana de Recife e Zona da Mata do Estado Pernambuco, Brasil.

Os suínos abatidos eram criados em 11 propriedades de sistema intensivo confinado, incluindo-se nesta categoria oito propriedades de ciclo completo com o sistema de integração com outras granjas, as quais foram denominadas granjas tecnificadas, e três propriedades de subsistência que criam suínos em pequena quantidade para o consumo próprio e fornecimento dos mercados de médio e pequeno porte, além das feiras livres (Miele; WaquiL, 2007). Para efeito de análise epidemiológica, as propriedades 
de subsistência foram computadas como um único grupo por apresentarem as mesmas características em relação às variáveis analisadas. Foi aplicado um questionário investigativo nas propriedades que abateram animais nos abatedouros, permitindo-se conhecer a identificação do produtor, identificação da propriedade, manejo higiênico-sanitário, além do acesso ao mercado e condição do plantel. Analisaram-se os abates ocorridos no período de julho de 2008 a maio de 2009 , totalizando 447 animais, machos e fêmeas, de raças variadas, em idade de abate, com peso vivo variando entre $80 \mathrm{a} 120 \mathrm{~kg}$.

Após o descanso, jejum anteabate einspeção antemortem, os animais eram encaminhados para a sala de matança. Na respectiva linha de inspeção foram observados, visualmentee por palpação, o estômago, intestino delgado e grosso, anotando-se as alterações em fichas previamente preparadas. Paralelamente, na própria linha de inspeção, realizou-se coleta de amostras fecais diretamente da ampola retal de cada animal, embaladas individualmente e mantidas refrigeradas até transporte ao laboratório.

As amostras fecais foram processadas no Laboratório de Doenças Parasitárias dos Animais Domésticos no Departamento de Medicina Veterinária da Universidade de Federal Rural de Pernambuco, por meio da contagem de ovos nas fezes (OPG) conforme GoRDON; WHITLOCK (1939) e coprocultura (ROBERTS; O'sULLIVAN, 1950) para a identificação delarvas de terceiro estágio de nematoides gastrintestinais
(HONER, 1967). Das 447 amostras coletadas, apenas 349 foram submetidas à coprocultura devido à quantidade de fezes insuficiente para os dois exames. As amostras foram processadas num período máximo de $24 \mathrm{~h}$ a contar do momento da coleta, sendo utilizadas 4 gramas de fezes para a coprocultura.

Para análise dos dados foram obtidas distribuições absolutas e percentuais para a frequência de parasitismo ao OPG e coprocultura, além da utilização do teste Qui-quadrado de Pearson ou Exato de Fisher (quando as condições para utilização do teste Quiquadrado não foram verificadas) para verificação da associação com o tipo de suinocultura e as variáveis do questionário investigativo. O nível de significância utilizado na decisão dos testes estatísticos foi de $5,0 \%$. Os dados foram digitados na planilha Excel eo "software" estatístico utilizado para a obtenção dos cálculos estatísticos foi o SPSS (Statistical Package for the Social Sciences) na versão 15.

\section{RESULTADOS E DISCUSSÃO}

Obteve-se positividade de 6,5\% (29/447) para helmintos nas amostras fecais de suínos analisadas, considerando-se os dois tipos de exames realizados. Detectaram-se ao OPG 2,7\% (12/447) de amostras positivas, observando-se ovos tipo Strongyloidea e Trichuris sp. com percentuais de 2,5\% (11/447) e $0,2 \%$ (1/447), respectivamente.

Tabela 1 - Frequência absoluta (n) e relativa (\%) de helmintos em amostras fecais de suínos de abatedouros na Região Metropolitana de Recife e Zona da Mata do estado de Pernambuco, Brasil segundo o tipo de suinocultura.

\begin{tabular}{|c|c|c|c|c|c|}
\hline \multirow{3}{*}{ Helmintos } & \multicolumn{2}{|c|}{ Suinocultura } & \multirow[b]{2}{*}{ Grupo total } & \multirow{3}{*}{ Valor de p } & \multirow{3}{*}{ OR (IC a 95\%) } \\
\hline & Tecnificada & Subsistência & & & \\
\hline & $\mathrm{n}$ & $\%$ & $\%$ & & \\
\hline
\end{tabular}

\begin{tabular}{lcccccccc} 
Strongyloidea & & & & & & & \\
Positivo & 4 & 1,0 & 7 & 12,1 & 11 & 2,5 & $\mathrm{p}^{1}<0,001^{*}$ & $* *$ \\
Negativo & 385 & 99,0 & 51 & 87,9 & 436 & 97,5 & \\
\hline Total & 389 & 100,0 & 58 & 100,0 & 447 & 100,0 & \\
\hline
\end{tabular}

\begin{tabular}{lccccccc}
$\begin{array}{l}\text { Hyostrongylus sp. } \\
\text { Positivo } \\
\text { Negativo }\end{array}$ & 10 & 3,4 & 1 & 1,7 & 11 & 3,1 & $\mathrm{p}^{1}=0,699$ \\
\hline Total & 282 & 96,6 & 57 & 98,3 & 339 & 96,9 & \\
\hline
\end{tabular}

\begin{tabular}{lccccccc}
$\begin{array}{l}\text { Oesophagostomum spp. } \\
\text { Positivo }\end{array}$ & 0 & 0 & 8 & 13,8 & 8 & 2,3 & $\mathrm{p}^{1}<0,001^{*}$ \\
Negativo & 292 & 100,0 & 50 & 86,2 & 342 & 97,7 & \\
\hline Total & 292 & 100,0 & 58 & 100,0 & 350 & 100,0 & \\
\hline
\end{tabular}

$\left.{ }^{*}\right)$ : Diferença significativa a 5,0\%.

$\left.{ }^{* *}\right)$ : Não foi determinado devido à ocorrência de intervalo muito amplo.

$\left({ }^{* * *}\right)$ : Não foi possível determinar devido à ocorrência de frequências nulas e muito baixas.

${ }^{1}$ Através do teste Exato de Fisher. 
Observou-se nas granjas tecnificadas OPG de 100, enquanto nas granjas de subsistência houve variação de 100 a 1200. Comparando-se a situação do parasitismo gastrintestinal nos dois tipos de fazendas pode-se observar que, apesar do maior número de amostras provenientes das granjas tecnificadas, o maior percentual de positividade pertenceu à granja de subsistência.

Hoff et al. (2005), no Estado de Santa Catarina, demonstraram resultados superiores aos encontrados neste estudo, na realização de exames coprológicos em amostras de fezes de suínos provenientes de três granjas de sistema confinado, revelando $21,5 \%(43 / 200)$ para ovos tipo Strongyloidea; $2 \%$ (4/200) para Ascaris suum e 0,5\% $(1 / 200)$ para Trichuris suis. Essa diferença pode ser devida à idade dos animais analisados; no estudo do referido autor, a maior infecção por ovos tipo Strongyloidea ocorreu em animais na fase de crescimento, sendo assim animais mais jovens que os do presente estudo, portanto mais susceptíveis ao parasitismo (Toma et al, . 2003). Lourensz (2003), no Vietnã, identificou ovos tipo Strongyloidea, $T$. suis e A. suum em, aproximadamente, 52\% (29/56)

das amostras examinadas.

Com relação às coproculturas, 5,5\% (19/349) foram positivas para helmintos, registrando-se $2,9 \%$ (10/349) para Hyostrongylus sp., 0,3\% (1/349) para Trichostrongylus sp. e 2,0\% (7/349) para o gênero Oesophagostomum, além de infecção mista por Hyostrongylus sp. e Oesophagostomum spp. em 0,3\% $(1 / 349)$ das amostras analisadas. Tais achados diferem dos relatados por RoEPSTORF; JORSAL (1989) na Dinamarca, que observaram $58 \%$ de Oesophagostomum spp.

No Estado dePernambuco, D'Alencaretal.(2006), analisando 1.065 amostras de suínos provenientes do Município de Camaragibe, identificaram os gêneros Hyostrongylus em 1,88\% (20/1065) e Trichostrongylus em $0,56 \%$ (6/1065), valores também baixos, próximos aosencontradosnestetrabalho.Ogênero Trichostrongylus raramente tem sido descrito dentre os helmintos gastrintestinais de suínos, no entanto, JESUS; MÜLLER (2000) registraram o encontro de helmintos adultos no estômago de suínos provenientes de criação doméstica da Região de Pelotas, RS.

Tabela 2- Frequência absoluta (n) e relativa (\%) de helmintos em amostras fecais de suínos de abatedouros da Região Metropolitana de Recife e Zona da Mata do Estado de Pernambuco, Brasil segundo as variáveis tipo de exploração, alimentação, assistência veterinária, estado nutricional, quarentena e comércio de animais vivos.

\begin{tabular}{|c|c|c|c|c|c|c|c|c|}
\hline \multirow{3}{*}{ Variáveis } & \multicolumn{4}{|c|}{ Helmintos } & & & \multirow{3}{*}{ Valor de $\mathrm{p}$} & \multirow{3}{*}{ OR (IC a 95\%) } \\
\hline & \multicolumn{2}{|c|}{ Positivo } & \multicolumn{2}{|c|}{ Negativo } & \multicolumn{2}{|c|}{ Total } & & \\
\hline & $\mathrm{n}$ & $\%$ & $\mathrm{n}$ & $\%$ & $\mathrm{n}$ & $\%$ & & \\
\hline \multicolumn{9}{|l|}{ Tipo de exploração } \\
\hline Recria & 0 & 0 & 39 & 100,0 & 39 & 100 & \multirow[t]{2}{*}{$\mathrm{p}^{(2)}=0,096$} & ** \\
\hline Mista & 29 & 7,1 & 379 & 92,9 & 408 & 100,0 & & \\
\hline \multicolumn{9}{|l|}{ Alimentação } \\
\hline Ração balanceada & 16 & 4,1 & 373 & 95,9 & 389 & 100,0 & \multirow[t]{2}{*}{$\mathrm{p}^{(1)}<0,001^{*}$} & 1,00 \\
\hline $\begin{array}{l}\text { Ração balanceada + restos de } \\
\text { alimentos }\end{array}$ & 13 & 22,4 & 45 & 77,6 & 58 & 100,0 & & $6,73(3,04$ a 14,91$)$ \\
\hline \multicolumn{9}{|l|}{ Assistência veterinária } \\
\hline Sim & 16 & 4,1 & 373 & 95,9 & 389 & 100,0 & \multirow{2}{*}{$\mathrm{p}^{(1)}<0,001^{*}$} & 1,00 \\
\hline Não & 13 & 22,4 & 45 & 77,6 & 58 & 100,0 & & $6,73(3,04$ a 14,91$)$ \\
\hline \multicolumn{9}{|l|}{ Estado nutricional } \\
\hline Bom & 28 & 6,9 & 378 & 93,1 & 406 & 100,0 & \multirow[t]{2}{*}{$\mathrm{p}^{(1)}=0,501$} & ** \\
\hline Moderado & 1 & 2,4 & 40 & 97,6 & 41 & 100,0 & & \\
\hline \multicolumn{9}{|l|}{ Quarentena } \\
\hline Sim & 16 & 4,1 & 373 & 95,9 & 389 & 100,0 & \multirow[t]{2}{*}{$\mathrm{p}^{(1)}<0,001^{*}$} & 1,00 \\
\hline Não & 13 & 22,4 & 45 & 77,6 & 58 & 100,0 & & $6,73(3,04$ a 14,91$)$ \\
\hline \multicolumn{9}{|l|}{ Comércio de animais vivos } \\
\hline Só no município & 13 & 22,4 & 45 & 77,6 & 58 & 100,0 & \multirow[t]{2}{*}{$\mathrm{p}^{(1)}<0,001^{*}$} & $6,73(3,04$ a 14,91$)$ \\
\hline Inter e intraestadual & 16 & 4,1 & 373 & 95,9 & 389 & 100,0 & & 1,00 \\
\hline Total & 29 & 6,5 & 418 & 93,5 & 447 & 100,0 & & \\
\hline
\end{tabular}

(*): Diferença significativa a 5,0\%.

$(* *)$ : Não foi determinado devido à ocorrência de intervalo muito amplo.

$\left.{ }^{* * *}\right)$ : Não foi possível determinar devido à ocorrência de frequências nulas e muito baixas.

(1): Através do teste Exato de Fisher.

(2) Através do teste do Qui-quadrado 
Comparando-se as taxas de infecção helmíntica com relação ao tipo de suinocultura (Tabela 1), observaram-se percentuais de positividade significativamente mais elevados entre os animais das granjas de subsistência do que entre os de granjas tecnificadas para ovos tipoStrongyloidea elarvas de Oesophagostomum spp., significando que, embora as criações de subsistência adotem um sistema intensivo de criação em que os animais são confinados, as condições de manejo adotadas, provavelmente, não são devidamente aplicadas, permitindo a sobrevivência de estágios infectantes e que os helmintos consigam completar o ciclo, frente ao manejo mais eficiente praticado nas granjas tecnificadas. A infecção por Oesophagostomum spp. nas granjas de subsistência foi confirmada ao exame das vísceras na linha de inspeção pela observação da presença de nódulos no intestino grosso (Fig. 1).

Nas Tabelas 2 a 4 registram-se os resultados obtidos das respostas ao questionário investigativo aplicado nas propriedades. Na Tabela 2, com exceção das questões "tipo deexploração" e "estado nutricional", para as demais variáveis comprova-se associação significativa $(p<0,05)$ com a ocorrência de helminto. Quanto à alimentação, nas oito propriedades tecnificadas fornecia-se apenas ração para os animais, enquanto na propriedade de subsistência além da ração, oferecia-se, também, restos alimentares, sendo a frequência de helmintos significativamente mais elevada neste último grupo, corroborando dados de KAGIRA et al. (2008), que relataram prevalência de helmintos significativamente maior em granjas que forneciam alimento não comercial. Associações significativas foram observadas também para assistência veterinária, realização de quarentena e comércio de animais (Tabela 2); convém atentar para o fato de que em todas estas variáveis os animais positivos correspondem as granja de subsistência. A utilização de quarentena como medida importante de biossegurança nas granjas foi registrada apenas nas granjas tecnificadas. Assim, os percentuais significativamente menores para os animais de granjas tecnificadas concordam com ROEPSTORFF; JORSAL (1989) que afirmaram que suínos em sistemas de produção altamente intensivos são infectados por nematoides em baixos níveis

Para variável comércio de animais vivos (Tabela 2), o mercado consumidor da suinocultura de subsistência são feiras livres dos próprios municípios onde se localizam os abatedouros, diferindo das oito propriedades do tipo de suinocultura tecnificada que fornecem animais a abatedouros da Região Metropolitana de Recife e também intra e interestadual. O comércio só no município corresponde à característica de subsistência da respectiva criação (Miele; WAQUiL 2007), desta forma, a associação significativa deve-se ao manejo instituído nas granjas de origem dos suínos comercializados.



Fig. 1 - Nódulos de Oesophagostomum spp. (setas) em intestino grosso de suíno de abatedouro 
Sobre o conhecimento dos entrevistados com relação à verminose, embora $77,8 \%(7 / 9)$ tenham respondido já terem ouvido falar sobre o assunto, houve associação significativa com a infecção helmíntica, com positividade significativamente maior em animais cujos responsáveis responderam não (Tabela 3). Por outro lado, em relação a manchas leitosas, todos os entrevistados das granjas tecnificadas desconheciam esta condição, e apenas 11,1\% (1/9) responderam positivamente, justamente suinocultores de subsistência, verificando-se, a despeito disso, percentual mais alto de infecção nos animais desta categoria de criação. Um fato para explicar esta situação pode ser o aparecimento frequente de tais lesões nos animais de subsistência abatidos, gerando o interesse em saber do que se trata, uma vez que existe o abate na própria propriedade para o consumo familiar ou mesmo para a comercialização informal neste tipo de criação. Como os animais de granjas tecnificadas geralmente vão para abatedouros, muitas vezes o proprietário não toma ciência da existência de tais lesões. Provavelmente, o não encontro de ovos nas fezes ou do parasito adulto deve-se ao fato de os animais estudados terem sido de idade adulta, cuja infecção já foi debelada, mas foram infectados em fases anteriores de sua vida.

Estes resultados demonstram que o pouco conhecimento sobre helmintos de suínos e de suas consequências para a suinocultura, o que impede o reconhecimento da necessidade da aplicação de medidas decontrole, contribuindo para a manutenção da infecção nos plantéis e para a ocorrência de prejuízos que são subestimados. De acordo com KanOra (2009), os sinais clínicos causados por helmintoses em suínos de engorda são, em sua maioria, vagos e inespecíficos. Consequentemente, suinocultores e veterinários consideram de baixa prioridade a importância econômica destas doenças por serem disponíveis informações inadequadas sobre seu impacto econômico, e porque atualmente há um interesse muito limitado, apenas em programas de controle inovadores.

Constatou-se o uso de anti-helmínticos em todas as granjas. Ao serem analisadas as bases químicas, observou-se o uso da ivermectina em 22,2\% (2/9) das granjas, sendo em uma delas utilizada uma formulação específica para suínos, fenbendazole em $66,7 \%$ (6/9) com uso contínuo na ração de forma profilática, além da ivermectina e cloridrato de levamisole em $11,1 \%$ (1/9). Analisando-se variáveis correspondentes ao controle das infecções helmínticas (Tabela 3), evidenciou-se associação significativa, com maior percentual $(18,2 \%)$ onde se utilizava como antihelmíntico ivermectina e cloridrato de levamisole, variando de $2,4 \%$ a $4,5 \%$ nas granjas que utilizavam outros produtos.

Tabela 3- Frequência absoluta (n) e relativa (\%) de helmintos em amostras fecais de suínos de abatedouros da Região Metropolitana de Recife e Zona da Mata do Estado de Pernambuco, Brasil segundo as variáveis conhecimento sobre verminose, manchas leitosas e controle anti-helmíntico.

\begin{tabular}{|c|c|c|c|c|c|c|c|c|}
\hline \multirow{3}{*}{ Variáveis } & \multicolumn{4}{|c|}{ Helmintos } & \multirow{2}{*}{\multicolumn{2}{|c|}{ Total }} & \multirow{3}{*}{ Valor de $\mathrm{p}$} & \multirow{3}{*}{ OR (IC a 95\%) } \\
\hline & \multicolumn{2}{|c|}{ Positivo } & \multicolumn{2}{|c|}{ Negativo } & & & & \\
\hline & $\mathrm{n}$ & $\%$ & $\mathrm{n}$ & $\%$ & \multirow[t]{2}{*}{$\mathrm{n}$} & \multirow{2}{*}{$\%$} & & \\
\hline \multicolumn{7}{|l|}{ Ouviu falar de verminose } & & \\
\hline Sim & 15 & 4,3 & 333 & 95,7 & 348 & 100,0 & \multirow{2}{*}{$\mathrm{p}^{(1)}<0,001^{*}$} & 1,00 \\
\hline Não & 14 & 14,1 & 85 & 85,9 & 99 & 100,0 & & $3,66(1,70$ a 7,87$)$ \\
\hline \multicolumn{8}{|l|}{ Ouviu falar de manchas de leite } & \\
\hline Sim & 13 & 22,4 & 45 & 77,6 & 58 & 100,0 & \multirow[t]{2}{*}{$\mathrm{p}^{(2)}<0,001^{*}$} & $\begin{array}{c}6,73(3,04 \mathrm{a} \\
1491)\end{array}$ \\
\hline Não & 16 & 4,1 & 373 & 95,9 & 389 & 100,0 & & 1,00 \\
\hline \multicolumn{9}{|l|}{ Anti-helmíntico } \\
\hline Ivomec & 1 & 2,4 & 40 & 97,6 & 41 & 100,0 & \multirow{4}{*}{$\mathrm{p}^{(1)}<0,001^{*}$} & ** \\
\hline Febendazole & 14 & 4,5 & 297 & 95,5 & 311 & 100,0 & & $* *$ \\
\hline Ivermectina para suínos & 1 & 2,7 & 36 & 97,3 & 37 & 100,0 & & $* *$ \\
\hline Ivomec + cloridrato delevamisol & 13 & 22,4 & 45 & 77,6 & 58 & 100,0 & & ** \\
\hline \multicolumn{9}{|l|}{ Rotação de anti-helmíntico } \\
\hline Sim & 29 & 7,1 & 379 & 92,9 & 408 & 100,0 & \multirow[t]{2}{*}{$\mathrm{p}^{(1)}=0,096$} & ** \\
\hline Não & 0 & 0 & 39 & 100,0 & 39 & 100,0 & & \\
\hline \multicolumn{9}{|l|}{ Exames coproparasitológicos } \\
\hline Sim & 1 & 2,7 & 36 & 97,3 & 37 & 100,0 & \multirow[t]{2}{*}{$\mathrm{p}^{(1)}=0,496$} & ** \\
\hline Não & 28 & 6,8 & 382 & 93,2 & 410 & 100,0 & & \\
\hline Total & 29 & 6,5 & 418 & 93,5 & 447 & 100,0 & & \\
\hline
\end{tabular}

(*): Diferença significativa a 5,0\%.

$\left({ }^{* *}\right)$ : Não foi determinado devido à ocorrência de intervalo muito amplo.

(1): Através do teste do Qui-quadrado

(2): Através do teste Exato de Fisher. 
Tabela 4 - Frequência absoluta (n) e relativa (\%) de helmintos em amostras fecais de suínos de abatedouros da Região Metropolitana de Recife eZona da Mata do Estado de Pernambuco, Brasil segundo as variáveis água, instalações e higiene.

\begin{tabular}{|c|c|c|c|c|c|c|c|c|}
\hline \multirow{3}{*}{ Variáveis } & \multicolumn{4}{|c|}{ Helmintos } & & & \multirow{3}{*}{ Valor de $p$} & \multirow{3}{*}{ OR (IC a 95\%) } \\
\hline & \multicolumn{2}{|c|}{ Positivo } & \multicolumn{2}{|c|}{ Negativo } & \multicolumn{2}{|c|}{ Total } & & \\
\hline & $\mathrm{n}$ & $\%$ & $\mathrm{n}$ & $\%$ & $\mathrm{n}$ & $\%$ & & \\
\hline \multicolumn{9}{|l|}{ Fonte de água } \\
\hline Poço & 15 & 4,8 & 298 & 95,2 & 313 & 100,0 & \multirow[t]{4}{*}{$\mathrm{p}^{2}<0,001^{*}$} & $* *$ \\
\hline Poço + açude & - & - & 39 & 100,0 & 39 & 100,0 & & \\
\hline Poço + rio & 1 & 2,7 & 36 & 97,3 & 37 & 100,0 & & \\
\hline Poço + barreiro & 13 & 22,4 & 45 & 77,6 & 58 & 100,0 & & \\
\hline \multicolumn{9}{|l|}{ Tratamento de água } \\
\hline Sim & 16 & 4,1 & 373 & 95,9 & 389 & 100,0 & \multirow[t]{2}{*}{$\mathrm{p}^{2}<0,001^{*}$} & 1,00 \\
\hline Não & 13 & 22,4 & 45 & 77,6 & 58 & 100,0 & & $6,73(3,04$ a 14,91$)$ \\
\hline \multicolumn{9}{|l|}{ Tipo de bebedouro } \\
\hline Chupeta & 16 & 4,1 & 373 & 95,9 & 389 & 100,0 & \multirow[t]{2}{*}{$\mathrm{p}^{2}<0,001^{*}$} & 1,00 \\
\hline Cocho & 13 & 22,4 & 45 & 77,6 & 58 & 100,0 & & $6,73(3,04$ a 14,91$)$ \\
\hline \multicolumn{9}{|c|}{ Qualidade de higiene das baias } \\
\hline Boa & 4 & 3,2 & 121 & 96,8 & 125 & 100,0 & \multirow[t]{3}{*}{$\mathrm{p}^{1}<0,001^{*}$} & 1,00 \\
\hline Moderada & 12 & 4,5 & 252 & 95,5 & 264 & 100,0 & & $1,44(0,46$ a 4,56$)$ \\
\hline Ruim & 13 & 22,4 & 45 & 77,6 & 58 & 100,0 & & $8,74(2,71$ a 28,21$)$ \\
\hline \multicolumn{9}{|l|}{ Ventilação } \\
\hline Boa & 20 & 6,0 & 314 & 94,0 & 334 & 100,0 & \multirow[t]{2}{*}{$\mathrm{p}^{1}=0,461$} & 1,00 \\
\hline Ruim & 9 & 8,0 & 104 & 92,0 & 113 & 100,0 & & $1,36(0,60$ a 3,08$)$ \\
\hline \multicolumn{9}{|l|}{ Tipo de piso } \\
\hline Áspero & 26 & 7,2 & 333 & 92,8 & 359 & 100,0 & \multirow[t]{2}{*}{$\mathrm{p}^{1}=0,191$} & $2,21(0,65$ a 7,48$)$ \\
\hline Liso & 3 & 3,4 & 85 & 96,6 & 88 & 100,0 & & 1,00 \\
\hline \multicolumn{9}{|l|}{ Limpeza das instalações } \\
\hline Jato d'água & 16 & 4,6 & 334 & 95,4 & 350 & 100,0 & \multirow[t]{2}{*}{$\mathrm{p}^{1}=0,002^{*}$} & 1,00 \\
\hline Remoção dos dejetos & 13 & 13,4 & 84 & 86,6 & 97 & 100,0 & & $3,23(1,50$ a 6,98$)$ \\
\hline \multicolumn{9}{|l|}{ Tratamento de dejetos } \\
\hline Sim & 5 & 3,0 & 161 & 97,0 & 166 & 100,0 & \multirow[t]{2}{*}{$\mathrm{p}^{1}=0,022^{*}$} & 1,00 \\
\hline Não & 24 & 8,5 & 257 & 91,5 & 281 & 100,0 & & $3,01(1,12$ a 8,04$)$ \\
\hline \multicolumn{9}{|l|}{ Destino dos dejetos } \\
\hline Fossa séptica & 2 & 2,6 & 76 & 97,4 & 78 & 100,0 & \multirow[t]{3}{*}{$\mathrm{p}^{1}=0,070$} & 1,00 \\
\hline Céu aberto & 24 & 8,5 & 257 & 91,5 & 281 & 100,0 & & $3,55(0,82$ a 15,36$)$ \\
\hline Lagoa de tratamento & 3 & 3,4 & 85 & 96,6 & 88 & 100,0 & & $1,34(0,22$ a 8,24$)$ \\
\hline Total & 29 & 6,5 & 418 & 93,5 & 447 & 100,0 & & \\
\hline
\end{tabular}

*Diferença significativa a 5,0\%.

**Não foi determinado devido à ocorrência de intervalo muito amplo.

***Não foi possível determinar devido à ocorrência de frequências nulas e muito baixas.

${ }^{1}$ Através do teste Qui-quadrado.

${ }^{2}$ Através do teste Exato de Fisher.

Nas granjas tecnificadas, o produtor, visando a produtividade, direciona suas ações de controle de forma a obter resultados seguros, favorecido também pelas condições econômicas que lhe permitem investir na implementação de um controle anti-helmíntico eficaz.

A permanência do parasitismo mesmo com o uso de anti-helmínticos concorda com estudo de KAGIRA et al. (2008), avaliando a prevalência de nematoides gastrintestinais com relação ao manejo em suínos no Kenya, em que anti-helmínticos foram utilizados em $97 \%$ das granjas, e as infecções por nematoides foram observadas em $94 \%$ delas, demonstrando que a maioria das granjas tem infecção patente.
Na Tabela 4 pode-se observar a existência de diferenças significativas entre as frequências de parasitismo para as variáveis: fonte de água, tratamento de água, tipo de bebedouro, qualidade de higiene das baias e limpeza das instalações. As frequências mais elevadas para cada uma destas variáveis $(18,2 \%)$ correspondem a animais de criações subsistência. Para a fonte de água, das propriedades analisadas, $66,7 \%(6 / 9)$ utilizavam água de poço; para as três restantes, as fontes foram poço e açude $(11,1 \%)$, poço e rio $(11,1 \%)$, poço e barreiro $(11,1 \%)$, nesta última não se fazia tratamento da água, nas demais (todas granjas tecnificadas), a água era tratada com cloro. Para o tipo de bebedouro, 88,9\% (8/9) das proprie- 
dades adotava bebedouro tipo chupeta e apenas $11,1 \%(1 / 9)$ cocho.

PIFFER etal. (1998) relataram três tipos de bebedouros: tipo taça, bebedouros em nível e os bebedouros tipo Nipple (chupeta); este último, utilizado nas oito propriedades de suinocultura tecnificada (Tabela 4), facilita a limpeza e o acesso dos animais e evita o desperdício de água. Seja qual for o modelo de bebedouro, é importante que o seu entorno seja desobstruído, para que os animais tenham maior facilidade de acesso e do ângulo de posicionamento adequado para a ingestão de água (PIFFER et al.,1998). A possibilidade da contaminação de água no cocho oferece um risco maior aos suínos, principalmente se no plantel existirem animais com a forma subclínica da doença.

A qualidade de higiene das baias foi considerada boa para 22,2\% (2/9) das granjas, moderada para $66,7 \%(6 / 9)$ e ruim para $11,1 \%(1 / 9)$, observando-se que os percentuais de parasitismo aumentaram à medida que a higiene das baias teve uma qualidade pior (Tabela 4). Em todas as granjas analisadas, a limpeza das instalações era realizada diariamente (apenas uma vez no dia), sendo realizada por meio de jato d'água em 77,8\% (7/9) das granjas e apenas remoção de dejetos em $22,2 \%$ delas (2/9), onde se obteve percentual maior significativamente maior. A prevalência e os graus de infecção helmíntica em suínos estão diretamente relacionados com a higiene e o sistema de manejo (RoepstorfF; Nilsson, 1991). KAGIRA et al. (2008) relataram prevalência geral da infecção por helmintos significativamente mais baixa nas granjas em que as fezes eram removidas diariamente, no entanto, além da remoção das fezes, segundo ROEPSTORFF (1991), a desinfecção dos tipos de piso reduz a ocorrência de suínos coprofágos, que são os maiores reservatórios da formas infectantes de vida livre dos parasitos e reduz a contaminação na granja. Nenhum tipo de desinfecção do piso das baias era praticado nas granjas analisadas além das medidas citadas.

Diferentemente de KAGIRA et al. (2008), que obtiveram prevalência significativamente maior de helmintos nas granjas que apresentaram piso de concreto quebrado, pedras, palha e pó de serra do que em granjas que apresentaram piso de concreto, não se observou associação significativa entre o parasitismo e o tipo de piso. A diferença entre os resultados pode ser devida ao fato de que, no presente estudo, todos os pisos eram de concreto, variando apenas em relação à porosidade (áspero e liso).

Outro fator importante está relacionado aos dejetos, verificando-se que em 33,3\% (3/9) das granjas realizava-se o tratamento dos dejetos; o destino era a fossa séptica em 22,2\% (2/9) e lagoa de tratamento em 11,1\% (1/9) (Tabela 4). Embora não se tenha obtido associação significativa neste estudo, convém salientar queéimportante a prática de se dar o destino adequado aos dejetos, evitando deixá-los expostos a insetos e roedores que podem servir de excelentes dispersores de parasitos. FöRSTER et al. (2009) demonstraram o potencial de Musca domestica carrear ovos e larvas de A. suum, T. suis e Metastrongylus sp., tanto no seu exoesqueleto quanto no intestino.

Apesar de o sistema de produção adotado em todas as granjas ser do tipo confinado, as condições sanitárias das propriedades de subsistência analisadas neste estudo favorecem a manutenção e a disseminação da infecção por helmintos gastrintestinais, sendo determinantes na prevalência e no grau de parasitismo mais elevados neste tipo de criação.

\section{REFERÊNCIAS}

BRASIL. Ministério do Agricultura e do Abastecimento. (RIISPOA). Departamento de Inspeção de Produtos de Origem Animal. Regulamento de Inspeção Industrial de Produtos de Origem Animal. Brasília/DF: DIPOA, 1997. p.190.

d'ALENCAR, A.S.; FAUSTINO, M.A.G.F.; SOUSA, D.P.; LIMA, M.M.; ALVES, L. C. Infecção por helmintos e coccídios em criação de suínos de sistema confinado localizada no município de Camaragibe-PE. Ciência Veterinária nos Trópicos, v.9, n. 2/3, p.79- 86, 2006.

FAO. Food and Agriculture Organization of United Nations. 2009. Disponível em: <http:www.fao.org.food> Acesso em: 4 de nov. 2009.

FÖRSTER, M.; KLIMPEL, S.; SIEVERT, K. The house fly (Musca domestica) as a potential vector of metazoan parasites caught in a pig-pen in Germany. Veterinary Parasitology, n.160, p.163-167, 2009 .

FORMIGA, D. N.; UENO, H.; LIGNON, G. B. Exames parasitológicos em amostras fecais de fêmeas suínas durante o ciclo reprodutivo. Concórdia: EMBRAPA/CNPSA, 1980. p.1-2. (Comunicado Técnico).

GORDON, H. Mcl.; WHITLOCK, H.V. A new technique for counting nematode eggs in sheep faeces. Journal Commowealth Science and Industry Organization, v.12, n.1, p.50-52, 1939.

HOFF, G.; SILVA, A.S.; MONTEIRO, S.G. Avaliação do parasitismo e comparação de técnicas de análise fecal em suínos de granjas da região oeste do estado de Santa Catarina. Revista da Faculdade de Zootecnia, Veterinária e Agronomia, v.12, n.1, p. 20-30, 2005.

HONER, M.R. The routine differentiation of the eggs and larvae of two parasites of swine, Hyostrongylus rubidus (Hassal et Stiles, 1882) and Oesophagostomum dentatum (Rud., 1803). Zeitschrift Fur Parasitenkunde, v.29, p.40-45, 1967 . 
IBGE. INSTITUTO BRASILEIRO DE GEOGRAFIA E ESTATÍSTICA. Produção da pecuária municipal. Anuário Estatístico do Brasil. v.35, 2007. Disponível em: <http:\\ www.ibge.gov.br.> Acesso em: 7 set. 2009.

JESUS, L.P.; MÜLLER, G. Helmintos parasitos de suínos na região de Pelotas, RS. Revista Brasileira de Agrociência, v.6, n.2, p.181-187, 2000.

KAGIRA, J.M.; KANYARI, P.W.N.; MUNYUA, W.K.; WARUIRU, R.M. Relationship between the prevalence of gastrointestinal nematode infections and management practices in pig herds in the Trika District, Kenya. Liverstock Research For Rural Development, v.20, n.10, 2008. Disponível em: $<$ http://www.lrrd.org/lrrd20/10/kagira20161.htm>. Acesso em: 10 out. 2009.

KANORA, A. Effect on productivity of treating fattening pigs every 5 weeks with flubendazole in feed. Vlaams Diergeneeskundig Tijdschrift, v.78, p.170-175, 2009.

KUNZ, A.; GIROTTO A.F.; MONTICELLI, C.J.; KICH, J.D.; FÁVERO, J.A.; LUDKE, J.V.; MORES, N., ABREU, P.G.; SILVEIRA, P.R.S. Produção de suínos. Concórdia: EMBRAPA/CNPSA, 2003. Disponível em: < http:www.famev. ufu.br/estrutura/disciplinas/documentos/apostilas_sps_embrapa_pdf $>$ Acesso em: 2 jun. 2009.

LEITE, D.M.G.; PEREIRA, N.W.; COSTA, A.O.D.; VARGAS, G.A.; SILVA, A. Parasitoses em suínos criados ao ar livre. Hora Veterinária, v.19, n.114, p.8-10, 2000.

LOURENSZ, J. Internal parasites of village pigs in hue province, Vietnam. Dookie College Institute of Land and Food Resources The University of Melbourne, Bachelor of Applied Science (Agriculture) Honours, Project in Agriculture 2003. 29p.

MIELE, M.; WAQUIL, P.D. Estrutura e dinâmica dos contratos na suinocultura de Santa Catarina: Um estudo de casos múltiplos. Estudos Econômicos, v.37, n.4, p.817-847, 2007.

MORA, L.M.O. Programas de desparasitación em porcino, valoración e eficácia. $A N A P O R C$, v.201, p.5-20, 2000.

PIFFER, I.A.; PERDOMO, C.C.; SOBESTIANSKY. Efeito de fatores ambientais na ocorrência de doenças. In: SOBESTIANSKY, J.; WENTZ, I.; SILVEIRA, P.R.S.; SESTI, L.A.C.
(Ed.). Suinocultura intensiva. Concórdia: Embrapa/CNPSA, 1998. cap. 13, p.261.

ROBERTS, F.H.S.; O'SULLIVAN, J.P. Methods for egg count and larval cultures for strongyles infesting the gastrointestinal tract of cattle. Australian Journal of Agricultural Research, v.1, p.99-102, 1950.

ROEPSTORFF, A. Transmission of intestinal helminths in Danish sow herds. Veterinary Parasitology, v.39, p.149-160, 1991.

ROEPSTORFF, A.; JORSAL, S. E. Prevalence of helminth infections in swine in Denmark. Institute of Internal Medicine, Royal Veterinary and Agricultural University, Frederiksberg C, Denmark. Veterinary Parasitology, v.33, n.4, p.231-239, 1989.

ROEPSTORFF, A; NILSSON, O. En faellesnordisk praevalensundersogelse. Países Baixos In: ERIKSEN, L; ROEPSTORFF, A; NANSEN, P. (Ed.). Parasitaere Infektioner hos Svin. NKJ. Project 59, Kobenhavn, 1991. p.16-53,

ROPPA, L. Suinocultura Brasileira. Suinocultura Industrial, v.20, n.134, p.24-32, 1998.

SILVA FILHA, O.L. Suinocultura local no Nordeste brasileiro. In: ENCUENTRO DE NUTRICIÓN Y PRODUCCIÓN EN ANIMALES MONOGÁSTRICOS, 9., 2007, Montevideo. Disponível em: $<$ http://www.fagro.edu.uy/ suinos/biblioteca/caract_socioecon/SE-Silva $\% 20 \% 20$ Suinocultura $\% 20$ local $\% 20$ no $\%$ nordeste $\% 20$ brasileiro.pdf.>. Acesso em: 27 out. 2009.

SONCINI, R.A.; MADUREIRA JÚNIOR, S.E. Monitorias Sanitárias. In: SOBESTIANSKY, J.; WENTZ, I.; SILVEIRA, R.R.S.; SESTI, A.C. (Ed.). Suinocultura intensiva: produção, manejo e saúde do rebanho. Brasília: Embrapa serviço de produção de informação, 1998. cap. 5, p.93-110.

TOMA, S.B.; MOREIRA, R.J.C.; CANAVACI, F.H.T. Atividade anti-helmíntica da ivermectina 1\% injetável em suínos naturalmente parasitados. Hora Veterinária, v.2, n.135, p.31-33, 2003.

Recebido em 6/4/10

Aceito em 22/4/11 